\title{
Gamified Consumer Engagement and Its Influence on Team Involvement over Time: An Abstract
}

\author{
Thilo Kunkel and Jason Doyle
}

\begin{abstract}
Gamified digital media offers many benefits to organizations. The purpose of this research project was to investigate the motives of consuming a gamified sport team app and its influence on team involvement over time. Drawing on involvement, engagement, and motivation theory, two studies were conducted. In Study 1, quantitative data of 639 users of a gamified mobile application was examined. Paired sample t-tests indicate a significant increase of app users' involvement mean score over the course of one soccer season. Subsequent linear regression analysis indicated a significant relationship between engagement related to generating knowledge (i.e., trivia questions) and the involvement difference between Time 1 and Time 2 explaining $6 \%$ of the variance of respondents' increased involvement. In Study 2, qualitative interviews with 27 users of the application revealed four themes representing consumers' motives for using the application - monetary rewards, competition, sense of achievement, and gaining knowledge. Additionally, the interviews explained the influence of the application on consumers' involvement changes or the lack of changes. This research contributes to consumer behavior knowledge by examining the intrinsic and extrinsic motives that drive the consumption of a gamified application and its influence on consumer involvement with an organization.
\end{abstract}

\footnotetext{
T. Kunkel $(\bowtie)$

Temple University, Philadelphia, PA, USA

e-mail: thilo.kunkel@temple.edu

J. Doyle

Griffith University, Brisbane, Australia

e-mail: jason.doyle@griffith.edu.au
} 